\title{
Caverna del Valle de Los Lisos: una hipótesis sobre su origen en el contexto geomorfológico de las Sierras de Córdoba, Argentina
}

\author{
Eduardo García-Aráoz ${ }^{1, *}$, Nicolás Madelón² y Marcela Cioccale ${ }^{3}$ \\ ${ }^{1}$ CONICET. Departamento de Geología Aplicada. FCEFyN, Universidad Nacional de Córdoba, \\ Av. Vélez Sarsfield 1611, Córdoba, C.P. 5000, Córdoba, Argentina. \\ ${ }^{2}$ Geólogo consultor. Benito Soria 345, Vélez Sarsfield 1611, Córdoba, C.P. 5000, Córdoba, Argentina. \\ ${ }^{3}$ Departamento de Geología Aplicada. FCEFyN, Universidad Nacional de Córdoba, \\ Av. Vélez Sarsfield 1611, Córdoba, C.P. 5000, Córdoba, Argentina. \\ *garciaaraoze@hotmail.com
}

\section{RESUMEN}

La caverna del Valle de Los Lisos conforma el cierre de una cuenca hidrográfica de aproximadamente 133 hectáreas, ubicada en el macizo Los Gigantes del batolito de Achala, en la Provincia de Córdoba, en la parte central de Argentina. Se compone de una serie de intrincados pasadizos desarrollados en litología granítica, con 20 metros de avance horizontal en sentido Norte-Sur y 25 metros de descenso total. Por su nivel inferior circula el arroyo que drena la cuenca. De acuerdo con las observaciones realizadas, se propone un modelo genético-evolutivo de la caverna que es consistente con la evolución geomorfológica regional de las Sierras de Córdoba, y consta de tres etapas luego del emplazamiento del cuerpo intrusivo: 1) Desarrollo de un perfil de meteorización profundo que condiciona la formación de un paleorelieve con predominio de una morfología abochonada. 2) A partir de un escarpe mixto (erosivo y estructural), la generación de la cuenca hidrográfica que alimenta el cauce que circula a través de la caverna. 3) Formación de las galerías principales en sucesivas fases de erosión.

Palabras clave: espeleología; rocas graníticas; geoformas; cueva.

\section{ABSTRACT}

The cavern of El Valle de Los Lisos is the closure of a watershed of approximately 133 hectares, located in Los Gigantes massif of the Achala batholith, Córdoba province, Argentina. This cavern is formed by a set of intricated passages developed in a granitic rock. The passages have 20 meters of horizontal advance in North-South direction, and 25 meters of total descent. The stream that drains the basin flows through its lower level.

According to the observations, a genetic-evolutionary model of the cavern is proposed, which is consistent with the Sierras de Córdoba regional geomorphological evolution. The model consists of three stages after the emplacement of intrusive body: 1) A deep weathering profile development that conditions the formation of a paleo-relief with a predominance of a rounded morphology;2) Hydrographic basin generation from an erosive and structural escarpment, which feeds the channel that circulates through the cave; 3) Main galleries formation in successive erosion phases.

Key words: speleology; granitic rocks; landforms; cave.

\section{INTRODUCCIÓN}

Los afloramientos graníticos presentan rasgos y características muy particulares, ya que en ellos coexisten formas heredadas y actuales que reflejan la evolución geomorfológica de la zona (Campbell, 1997; Campbell y Twidale, 1995; Twidale y Vidal-Romaní, 2005; Migon, 2006; Twidale, 2012). Cada uno de los elementos que componen este tipo de paisajes posee una historia que explica su origen. Esto permite que, a partir de su análisis, pueda obtenerse una propuesta evolutiva que relacione las morfologías en espacio y tiempo, reconociendo una superposición de las formas, siendo las menores, generalmente, las más modernas (y que siguen evolucionando en el presente) y las mayores las originadas bajo condiciones muy distintas a las actuales y que quedan como relictos de relieves antiguos (Vidal-Romaní y Yepes, 2004).

Tradicionalmente el estudio de las cavidades graníticas ha sido realizado desde dos enfoques diferentes: geomorfológico y espeleológico. Dentro del primero se destacan los trabajos de Sjöberg (1969), Finlayson (1981), Ollier y Smith (1994), Willems et al. (2002), Gaal y Bella (2008), Lazaridis y Pipera (2008), Roqué et al. (2011) y Song et al. (2011 y 2015) entre otros, orientados a realizar una descripción de los procesos observados y clasificar morfológica o genéticamente estos espacios. El segundo enfoque, en cambio, se orienta a una descripción más acabada de la arquitectura del ambiente subterráneo, sus dimensiones y relaciones espaciales (e. g.. Lazarte, 2002; Scheuerer et al., 2013). En el presente trabajo tenemos la intención de complementar estos enfoques en una propuesta integral que considere, además 
de los aspectos mencionados, el análisis del contexto histórico del macizo rocoso para explicar el origen y evolución de estos espacios.

Consecuentemente, se presentan aquí los resultados de un estudio geomorfológico-espeleológico realizado en la caverna del Valle de los Lisos, ubicada sobre el macizo de Los Gigantes en la Provincia de Córdoba, teniendo como criterios principales de análisis las geoformas que la componen, el contexto geológico en que se encuentra y los procesos que le dieron origen, en relación con la evolución geomorfológica del macizo granítico de Achala.

De acuerdo con la definición acuñada por Bates y Jackson (1987), se emplea el término "caverna" como referencia a una cueva de gran tamaño o un complejo de cuevas interconectadas, entendiéndose como "cueva" un espacio natural abierto bajo tierra, por lo general con una abertura en la superficie.

\section{UBICACIÓN Y MARCO GEOLÓGICO}

La caverna se encuentra ubicada en el Valle de los Lisos del macizo de Los Gigantes, perteneciente al batolito de Achala, en el sector central de las Sierras Grandes de Córdoba, en la Provincia de Córdoba, Argentina (Figura 1a). Se accede al lugar por un paraje de montaña situado sobre el camino que lleva al yacimiento uranífero Schlagintweit, a $28 \mathrm{~km}$ al W de la localidad de Tanti y a $70 \mathrm{~km}$ al W de la ciudad de Córdoba Capital.

Las sierras Grandes de Córdoba se componen de una serie de bloques de basamento, limitados por fallas inversas, que forman cordones montañosos de orientación meridiana (Carignano et al., 1999) (Figura 1a). Estos bloques presentan una suave inclinación al E y están truncados por cuatro superficies planas de erosión (Figura 1b), limitadas por escarpes compuestos (erosivos y estructurales) (Carignano et al., 2014).

Debido a su disposición escalonada mayormente sobre los 1,000 m s.n.m., estas superficies son comúnmente denominadas pampas de altura. Su morfología es suavemente ondulada, lo que ha permitido la acumulación de sedimentos post-pliocénicos que sólo han sido caracterizados en sitios puntuales (Montes, 1956; Manzur, 1995; Tauber y Goya, 2006; Carignano et al., 2014; Krapovickas y Tauber, 2016).

Krapovickas y Tauber (2016) sintetizan toda la información estratigráfica disponible de estas pampas de altura, en la columna generalizada de la Fm. Vaca Corral que tiene una edad ca. 37,095 (Optically Stimulated Luminescence) - 7,206 (Accelerator mass spectrometry) cal. años AP (Figura 2).

De acuerdo con Carignano et al. (1999 y 2014), la evolución geomorfológica general de las Sierras puede sintetizarse en tres eventos principales: a) Un prolongado período de estabilidad durante el Mesozoico inferior y medio con generación del etchplain bajo condiciones climáticas húmedas y cálidas. b) Erosión y desarrollo de pedimentos y escarpes asociados a un cambio del nivel de base por procesos distensivos (rifting), sumados a un clima progresivamente más seco (Cretácico). c) Alzamiento general de la Sierra originada por la tectónica andina (Cenozoico) e instalación de las condiciones climáticas actuales.

Dentro de este contexto general, el Batolito de Achala se presenta como un macizo granítico de geometría marcadamente elíptica, con una superficie aproximada de $2,500 \mathrm{~km}^{2}$ (Figura 1a), emplazado con rumbo submeridional NNE $\left(20^{\circ}\right)$ en las metamorfitas de grado medioalto del basamento cristalino de la Sierra Grande de Córdoba (Gordillo y Lencinas, 1979). La edad de cristalización es Devónico Tardío (379 \pm 4 Ma, U-Pb en circón, SHRIMP, Rapela et al., 2008).

De acuerdo con Martino et al. (2017), pueden diferenciarse dos grandes dominios geomorfológicos con rasgos estructurales distintivos dentro del Batolito de Achala: el Dominio Achala Norte (DAN) que se caracteriza por un relieve granítico suave y el Dominio Achala Sur (DAS) que presenta un relieve granítico escarpado. Ambos dominios están separados por una falla transtensional dextral, de rumbo noroeste y casi vertical llamada lineamiento Retamito (RL) (Figura 1a) (Martino et al., 2012b y 2017). Este lineamiento eleva el bloque granítico del cerro Los Gigantes (2,400 m s.n.m) produciendo el basculamiento hacia el norte del bloque del DAN y hacia el este del bloque del DAS.

Dentro del DAN, se reconocen una serie de lineamientos que forman profundos valles en $\mathrm{V}$ y delimitan bloques alargados con rumbo NW. Estos lineamientos son denominados de norte a sur (Figura 1a): Candelaria (CL), Corral del Carnero (CCL), Rincón Grande (RGL), Guasta (GL) y Retamito (RL) (Martino et al., 2012b y 2017).

Por su parte, el DAS está subdividido por una serie de fallas paralelas de rumbo N, denominadas de oeste a este (Figura 1a): fallas Rugapampa (RU), Río Hondo (RH), Potrero de Gero (PG), Cumbre de Achala (CA), Boca del Río (BC) y La Cumbrecita (LC), que producen un escalonamiento progresivo hacia el centro de la unidad, (Martino et al., 2017).

Respecto a las condiciones climáticas del sector de estudio, las precipitaciones medias anuales oscilan entre los 900 y 1,000 mm y se concentran entre los meses de noviembre y marzo, mientras la temperatura media anual es de $10^{\circ} \mathrm{C}$, con temperaturas máximas de 25 a $30^{\circ} \mathrm{C}$ y mínimas de -10 a $-25^{\circ} \mathrm{C}$ (Capitanelli, 1979).

\section{DESCRIPCIÓN DEL ENTORNO}

El Valle de los Lisos conforma una cuenca hidrográfica de geometría elongada en sentido N-S con una superficie aproximada de 133 hectáreas (Figura 1a). Esta cuenca presenta la particularidad de no poseer una desembocadura de drenaje superficial, sino que su cierre hidrográfico se realiza a partir de la infiltración del cauce principal entre los bloques y sedimentos que componen el fondo de un pequeño valle que antecede a la caverna.

En ese sector, la cobertura sedimentaria está formada por una secuencia de materiales arcillo-limosos de tonalidades oscuras, intercalados con depósitos lenticulares de arenas medias y finas de poco espesor (Figura 3). Su granulometría, disposición y contenido de materia orgánica denotan un ambiente de muy baja energía y bajos niveles de oxígeno (sedimentos finos de tonalidad oscura) con episodios esporádicos de mayor energía (arenas). Se trata de sedimentos desarrollados en ambientes palustres/lacustres intercalados con eventos de acumulación eólica correlacionables con las facies superiores fluvio/ loéssicas de la columna generalizada de Krapovickas y Tauber (2016). Por encima de estos depósitos se desarrolla el suelo actual.

En contraste, las zonas más altas de la cuenca poseen una escasa cobertura sedimentaria, generalmente formada por granito meteorizado (regolito), con poco desarrollado edáfico.

El tamaño de los bloques observados en el entorno es variable y en general ostentan dimensiones que exceden la capacidad de carga del arroyo (de 0.5 a $5 \mathrm{~m}$ de diámetro). Presentan una geometría prismática desarrollada por la intersección de tres familias de discontinuidades principales $\left(9^{\circ} / 56^{\circ} \mathrm{W}, 16^{\circ} / 48^{\circ} \mathrm{E}, 112^{\circ} / 87^{\circ} \mathrm{N}\right)$ (Figura 4 ).

Estos mismos patrones de fracturamiento han sido reconocidos también en el interior de la caverna, manifestando un fuerte control estructural en el desarrollo de sus galerías (Figura 4).

Además de estas estructuras, se reconocen diferentes geoformas graníticas (Figura 5): bloques, cuñas, acanaladuras, pilas, roturas poligonales, tafoni y superficies zapadas; todas ellas, en conjunto, evidencian una historia geomorfológica del macizo que se inicia durante los estadios magmáticos y se sucede, al menos, en dos etapas posteriores (Carignano et al., 1999; Twidale y Vidal-Romaní, 2005; Migon, 2006; 

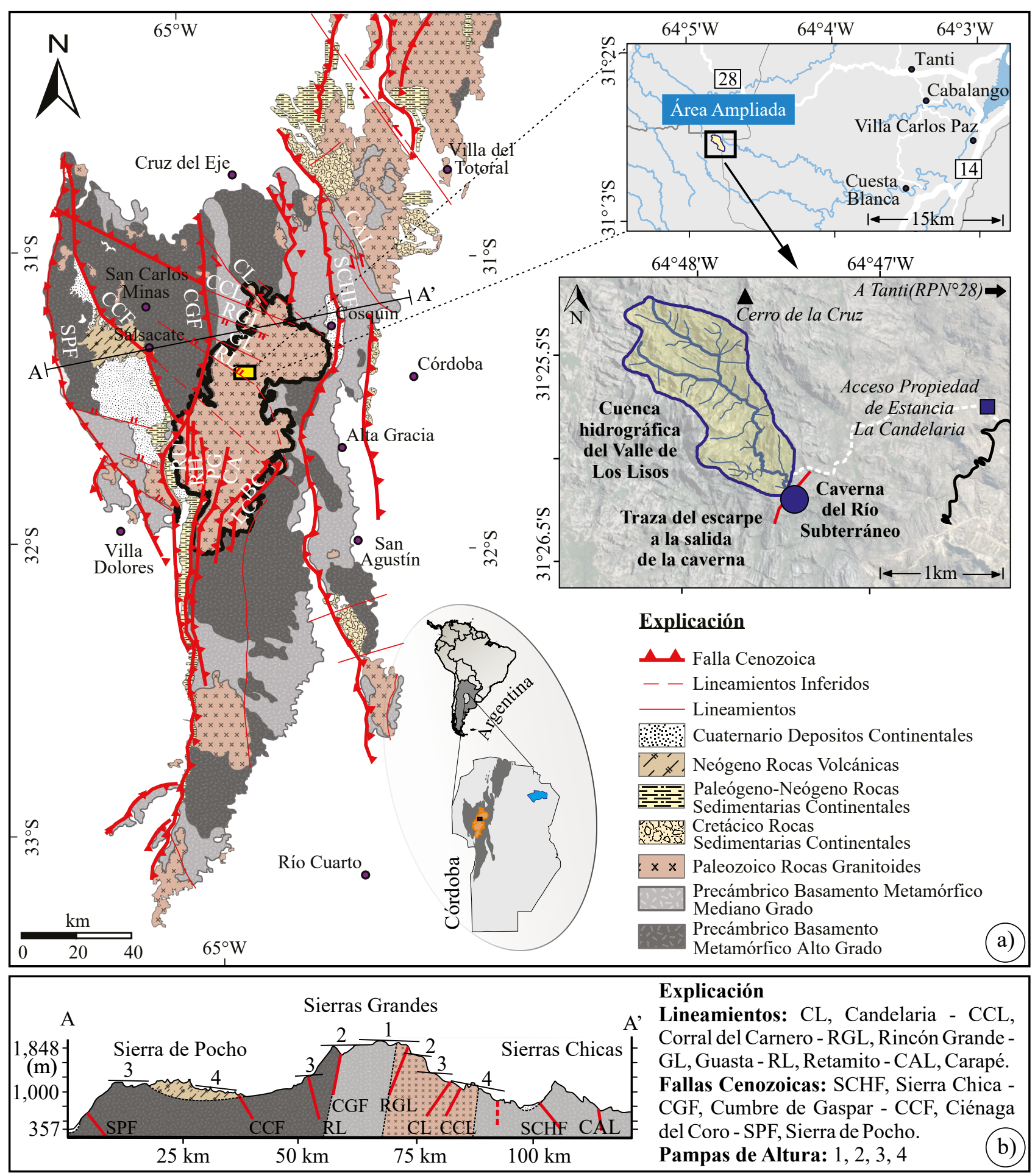

Figura 1. a) Mapa geológico de las Sierras de Córdoba y ubicación del sitio de estudio (coordenadas geográficas de la caverna: $31.436169^{\circ} \mathrm{S} / 64.791469^{\circ} \mathrm{W}$ ). Lineamientos: Carapé (CAL) Candelaria (CL), Corral del Carnero (CCL), Rincón Grande (RGL), Guasta (GL) y Retamito (RL). Fallas cenozoicas: Sierra Chica (SCHF), Cumbre de Gaspar (CGF), Ciénaga del Coro (CCF), Sierra de Pocho (SPF) Rugapampa (RU), Río Hondo (RH), Potrero de Gero (PG), Cumbre de Achala (CA), Boca del Río (BC) y La Cumbrecita (LC) (Modificado de Martino et al., 2012a). b) Corte estructural de las Sierras de Córdoba, mostrando las principales pampas de alturas: 1, 2, 3 y 4 (Modificado de Carignano et al., 1999). 


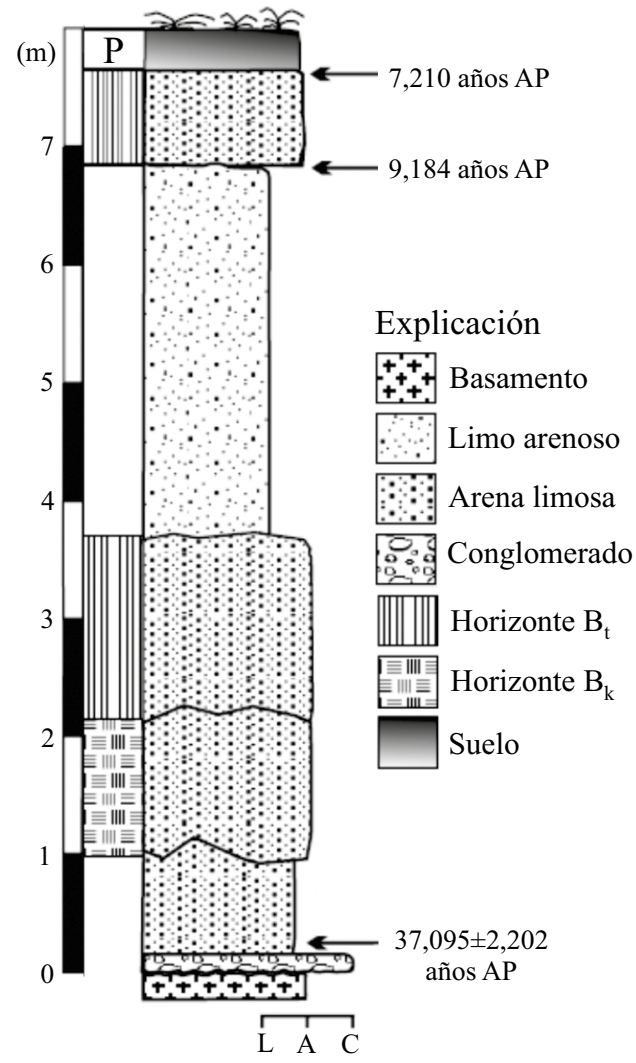

Figura 2. Columna Generalizada de la Fm. Vaca Corral para las pampas de altura de las Sierras Pampeanas de Córdoba (modificada de Krapovickas y Tauber, 2016). Se identifican cinco facies sedimentarias de base a techo: 1- Limo arenoso con megatabiques de carbonato de calcio, 2- Arena limosa pardo rojiza con clastos, 3- Arena limosa laminada y cementada, 4- Limo arenoso amarillento y 5- Arena limosa oscura maciza. P: horizontes pedogenéticos inferidos. $\mathrm{B}_{\mathrm{t}}$ : Horizonte $\mathrm{B}$ con arcillas. $\mathrm{B}_{\mathrm{k}}$ : Horizonte B con carbonatos. L: Limo. A: Arena. C: Conglomerado.

Twidale, 2012): una etapa de intensa y prolongada meteorización química, y una etapa predominantemente erosiva donde se definen las formas encontradas.

\section{RELEVAMIENTO DE LA CAVERNA}

La caverna se compone de una serie de intrincados pasadizos desarrollados en litología granítica. Si bien muestra una menor extensión comparada con otras cavernas graníticas típicas del mundo, tales como Labertourche en Victoria (Finlayson, 1981) o Crombie's Cave en Nueva Gales (Ollier y Smith, 1994), es una de las de mayor desarrollo dentro del territorio nacional argentino. La caverna presenta 20 metros de avance horizontal en sentido N-S y 25 metros de descenso total (Figura 6a y 6b). Se han identificado tres aberturas de ingreso y dos de salida en diferentes niveles topográficos con evidentes signos de erosión hídrica. Actualmente el arroyo circula a través del nivel más profundo, conectando la entrada y la salida inferior por medio de pequeños conductos intransitables.

Es común, en todos los corredores, la presencia de bloques de diversos tamaños, algunos redondeados, otros irregulares, que se traban entre si logrando un equilibrio mecánicamente estable. Estos bloques presentan, en sus zonas de contacto, depósitos remanentes de los pulsos de crecidas (ramas, troncos, restos vegetales, etc.).
Dado que las galerías de acceso conforman caminos individuales que confluyen en un punto, la arquitectura del espacio subterráneo se resuelve en dos sectores con características diferentes. Un sector Norte, aguas arriba del punto de confluencia y un sector Sur por debajo de ese punto. El sector norte se caracteriza por presentar galerías estrechas, en general con forma tabular de entre 0.05 a $1 \mathrm{~m}$ de ancho, definidas entre los planos de fractura con bloques menores alojados (Figura $6 \mathrm{c}$ y 6e). En algunos sectores las galerías se ciñen de manera tal que se dificulta bastante el acceso. Hacia el sur, en cambio, cuando se unen los diferentes caminos de ingreso, el espacio es más amplio, llegando a quedar definidas recámaras de hasta 2 a $3 \mathrm{~m}$ de ancho con similar desarrollo vertical (Figura 6d). En este sector son importantes los signos de erosión hídrica abrasiva, las rocas en general presentan un pulido superficial y son comunes los socavones y las marmitas (Figura 6f).

Las salidas de la caverna limitan con un escarpe de pendiente abrupta. Los numerosos bloques que se observan al pie de este escarpe son una evidencia directa de un proceso de retroceso erosivo que está actuando sobre el mismo (Figura 5).

Un rasgo particular que se ha identificado en diferentes sectores de la cavidad es la presencia de espeleotemas silíceas, que constituyen formas de acumulación en el ambiente subterráneo dominado por procesos erosivos. Hallazgos similares son reportados por Cioccale $e t$ al. (2008) y Vidal-Romaní et al. (2010) en otras zonas del batolito de Achala. Las espeleotemas más abundantes son de tipo coraloides (de acuerdo con la clasificación de Cioccale et al., 2008) y se desarrollan fundamentalmente en las intersecciones de dos o más fracturas, formando estructuras irregulares (semejantes a corales ramiformes) de entre 3 a $15 \mathrm{~mm}$ de largo, con textura porosa y color pardo a pardo amarillento.

\section{GÉNESIS Y PROPUESTA EVOLUTIVA PARA LA CAVERNA}

La propuesta evolutiva que se presenta a continuación ha sido concebida con base en una serie de observaciones y características particulares que se reconocieron en el sitio de estudio:

La caverna se forma por una corriente que funciona de manera subterránea en un área del granito intensamente diaclasada.

La orientación consistente de los bloques mayores muestra que el macizo está esencialmente en su sitio.

La zona que antecede a la caverna conforma una depresión con forma de cubeta, rellena con sedimentos finos (interpretados como de origen lacustres) que sugieren periodos de anegamiento en el sector.

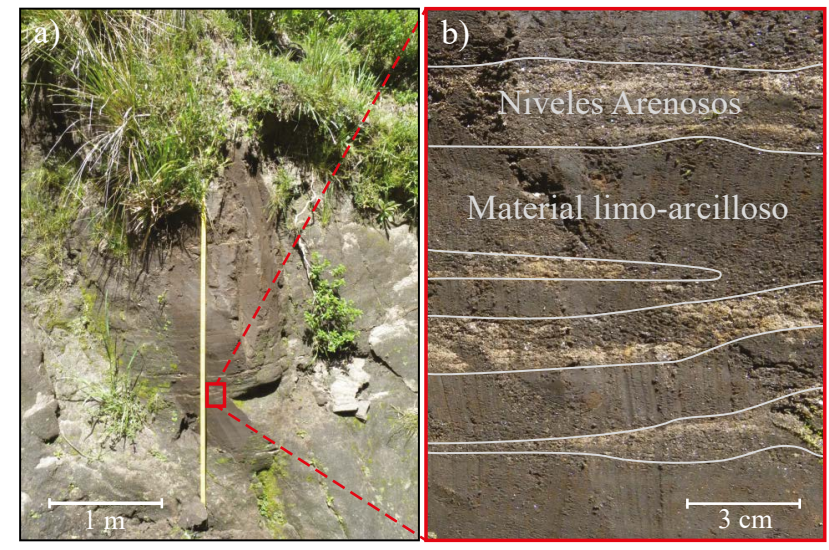

Figura 3. Sedimentos lacustres. a) Vista general del afloramiento. b) Vista en detalle. 


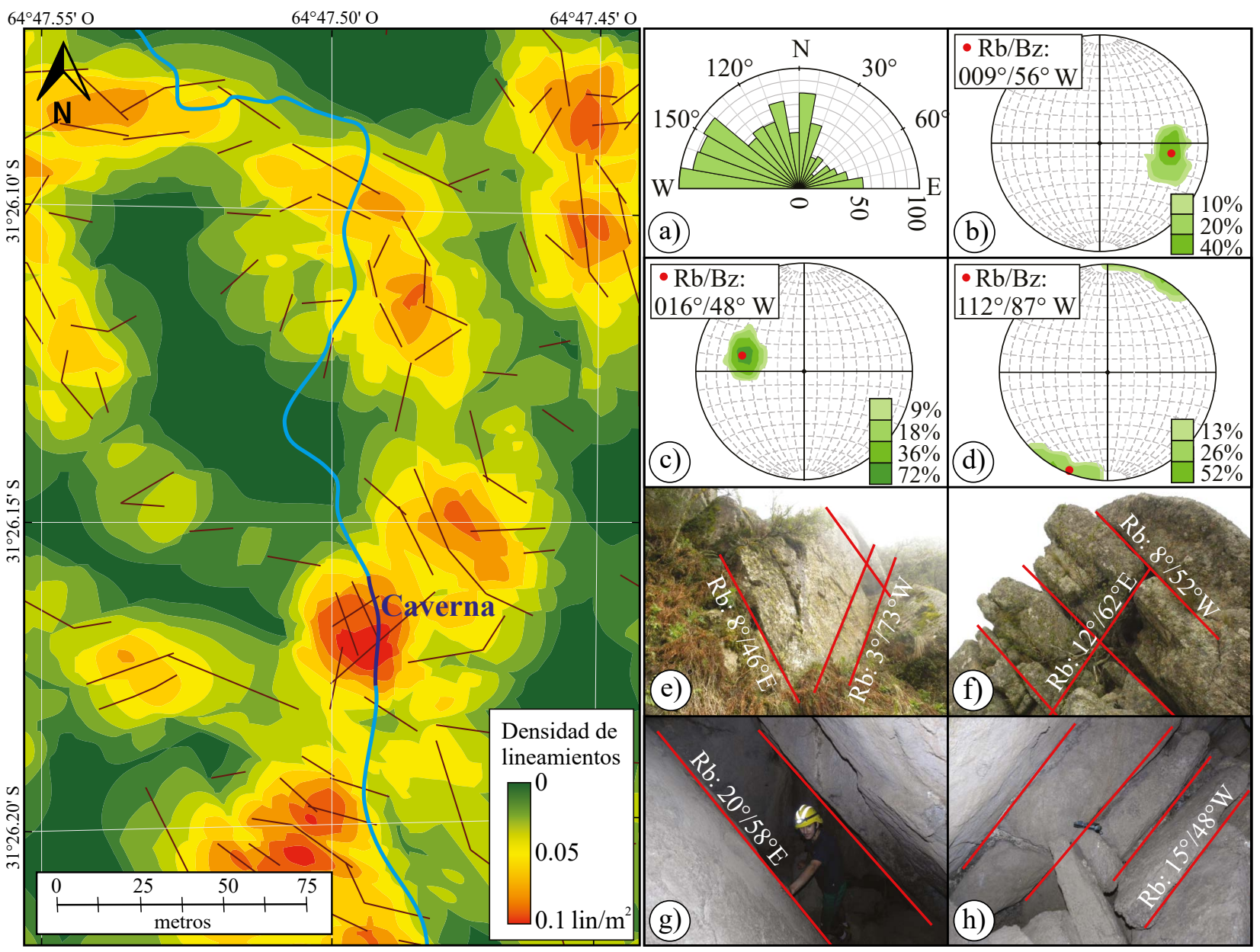

Figura 4. Mapa de densidad de lineamientos (cantidad de lineamientos por metro cuadrado) identificados en imagen satelital (Orbview). a) Diagrama de rosa de frecuencia de orientaciones del mapa de lineamientos. b), c) y d) Proyección estereográfica de las tres familias de fracturas identificadas y cartografiadas en campo. Los polos se grafican en el hemisferio inferior con sus valores de Rumbo ( $\mathrm{Rb}$ ) y Buzamiento (Bz) promedio. e) y f) Fracturas afectando el granito aflorante en los alrededores de la caverna. g) y h) Fracturas en el espacio subterráneo.

La disposición de las entradas y salidas en distintos niveles topográficos indica una evolución en sucesivas fases erosivas desde los niveles superiores a los inferiores de la caverna. La presencia de superficies zapadas en las inmediaciones favorece esta interpretación.

Las galerías del sector Norte de la caverna presentan secciones muy angulares, dominadas por discontinuidades poco modificadas. La preservación de planos angulares en las discontinuidades indica que el proceso de pre-meteorización no fue tan extenso y/o que estamos en presencia de una etapa temprana de cavernamiento.

Las galerías del sector Sur se encuentran más redondeadas, siendo comunes los socavones y superficies pulidas cóncavas formadas por abrasión de la corriente subterránea. Esto indica que el río no sólo ha bajado su curso por erosión del material alterado a través de las discontinuidades, sino que además, ha atravesado roca sólida por erosión mecánica abrasiva.

La posición de la caverna adyacente a un escarpe pronunciado es propicia para la incisión vertical del cauce y la formación del espacio subterráneo.

En la Figura 7 se presenta el modelo esquemático que resume el desarrollo evolutivo de la caverna y contempla tres etapas luego del emplazamiento del cuerpo intrusivo:

La primera etapa (Figura 7a) corresponde a un periodo de estabilidad tectónica, donde se desarrolla un profundo perfil de meteorización que condiciona la formación de un paleorrelieve (etchplain) con predominio de una morfología abochonada y asociaciones de geoformas graníticas características.

La segunda etapa (Figura $7 \mathrm{~b}$ ) se produce con la generación del resalto topográfico que se observa a la salida de la caverna. Se trata de un escarpe mixto producto de procesos coluviales y tectónicos.

Esta nueva configuración del relieve generaría la formación de la cuenca hidrográfica del Valle de Los Lisos. En las etapas iniciales de este sistema de drenaje, la zona más deprimida de la cuenca (donde se ubica actualmente la caverna), habría operado como lugar de encharcamiento durante las estaciones húmedas. $\mathrm{El}$ anegamiento recurrente aumentaría la infiltración a través de las discontinuidades del macizo, produciendo una mayor alteración de las paredes e incrementando el material disponible para erosión.

La tercera etapa del modelo propuesto contempla la mayor parte de la generación del espacio subterráneo. Las Figuras 7c, 7d, 7e y 7f, ilustran las sucesivas fases de formación de las galerías principales.

Cada conducto subterráneo ha sido formado por la acción erosiva del agua que remueve el material previamente alterado. Cada vez que se libera una nueva vía de circulación subterránea se produce un descenso relativo del nivel de base del río y, en consecuencia, un aumento de los procesos erosivos. Como resultado de este mecanismo, a medida que es removido el material disponible para erosión, 


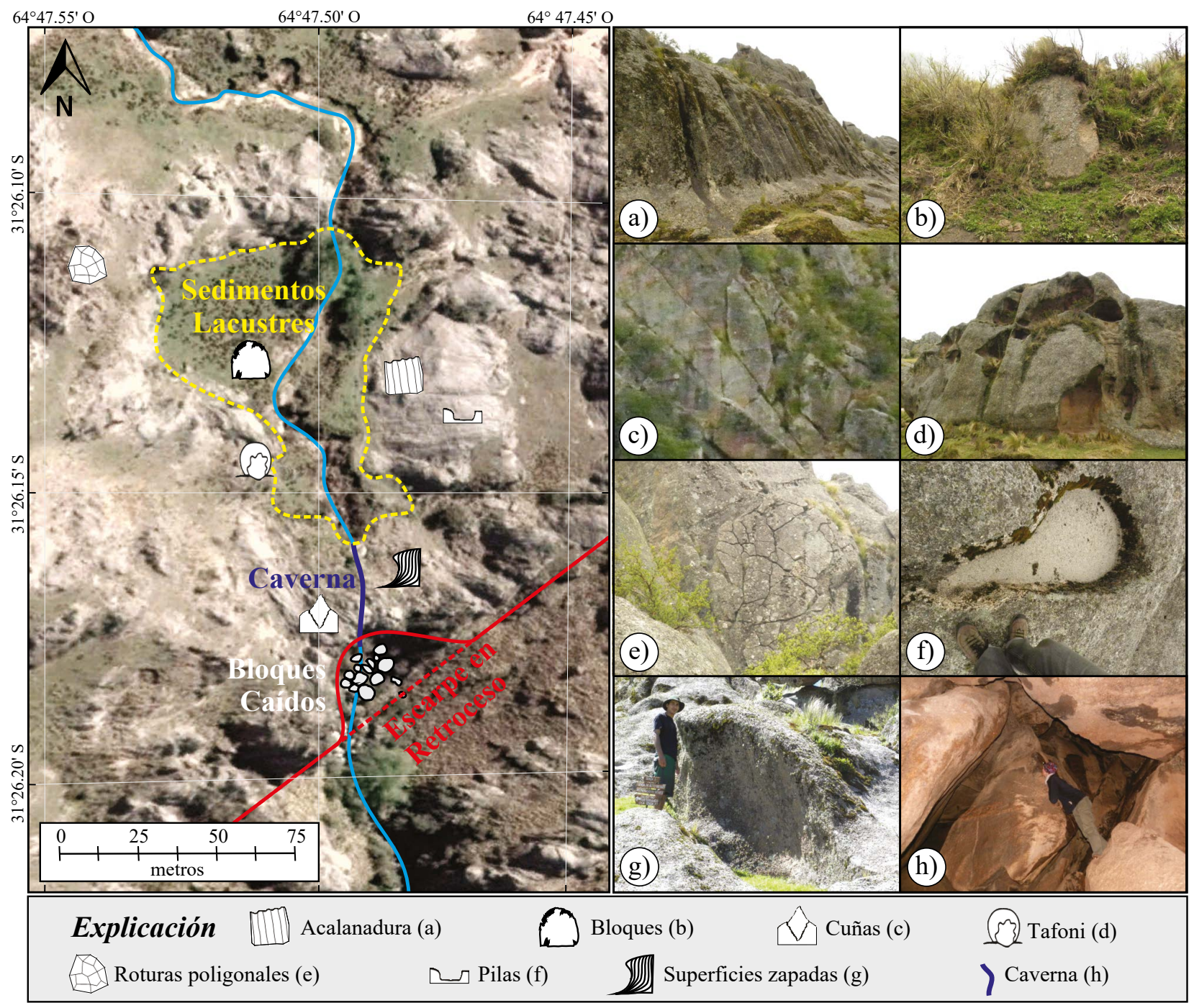

Figura 5. Entorno geomorfológico de la caverna. Las referencias indican sobre el mapa el tipo de geoforma encontrada y las imágenes a) a h), muestran una vista de cada una de ellas.

se produce también un reacomodamiento gravitatorio de los bloques suprayacentes, trabándose unos con otros y dejando diversos espacios vacíos entre ellos. Finalmente, la caverna se forma por la coalición de estos espacios vacíos.

Este proceso de apertura de nuevos conductos subterráneos puede iniciarse por dos motivos: 1) una obstrucción del circuito hidrogeológico instaurado que obliga al agua a encontrar otro camino, y/o 2) por erosión durante un episodio de crecida, donde se ve excedido el curso original.

De esta manera, y de acuerdo con el modelo propuesto, la construcción del espacio subterráneo se realiza en sucesivas fases erosivas desde los niveles superiores a los inferiores.

\section{DISCUSIÓN}

Los eventos principales que Carignano et al. (1999, 2014) consideran determinantes en la evolución general de las Sierras de Córdoba han sido reconocidos también en diferentes estudios puntuales sobre los paisajes graníticos del Batolito de Achala. Particularmente, las contribuciones de Cerutti (2002), García-Aráoz y Cioccale (2015) y Crespi (2015), señalan que es posible determinar una sucesión cronológica en las geoformas observadas, permitiendo reconstruir una historia geomorfológica del macizo coherente con estos eventos.

De acuerdo con estos autores, la historia geológica de estas geoformas comenzaría con la intrusión del batolito hace aproximadamente $367 \mathrm{Ma}$ (Dorais, et al., 1997). En estos primeros estadios se generarían estructuras tales como flujos, foliaciones magmáticas y agrietamiento poligonal (que terminaría de desarrollarse y sería expuesto mucho después; Vidal-Romaní y Yepes, 2004).

Hace, por lo menos, $120 \mathrm{Ma}$ (Lobens et al., 2011; Bense et al., 2013) la Pampa de Achala correspondería a un elemento positivo, constituido por rocas graníticas afectadas por diaclasas ortogonales, a través de las cuales los procesos de meteorización química (bajo un clima templado húmedo a subtropical) habrían actuado durante un largo periodo de estabilidad tectónica para producir el etchplain Jurásico (Carignano et al., 1999, 2014). El frente de meteorización se propagaría en forma diferencial, controlado por la densidad de fracturas y las características diferenciales del propio granito.

Luego, hace aproximadamente $80 \mathrm{Ma}$ (Lobens et al., 2011; Bense et al., 2013), los profundos perfiles de meteorización generados serían 

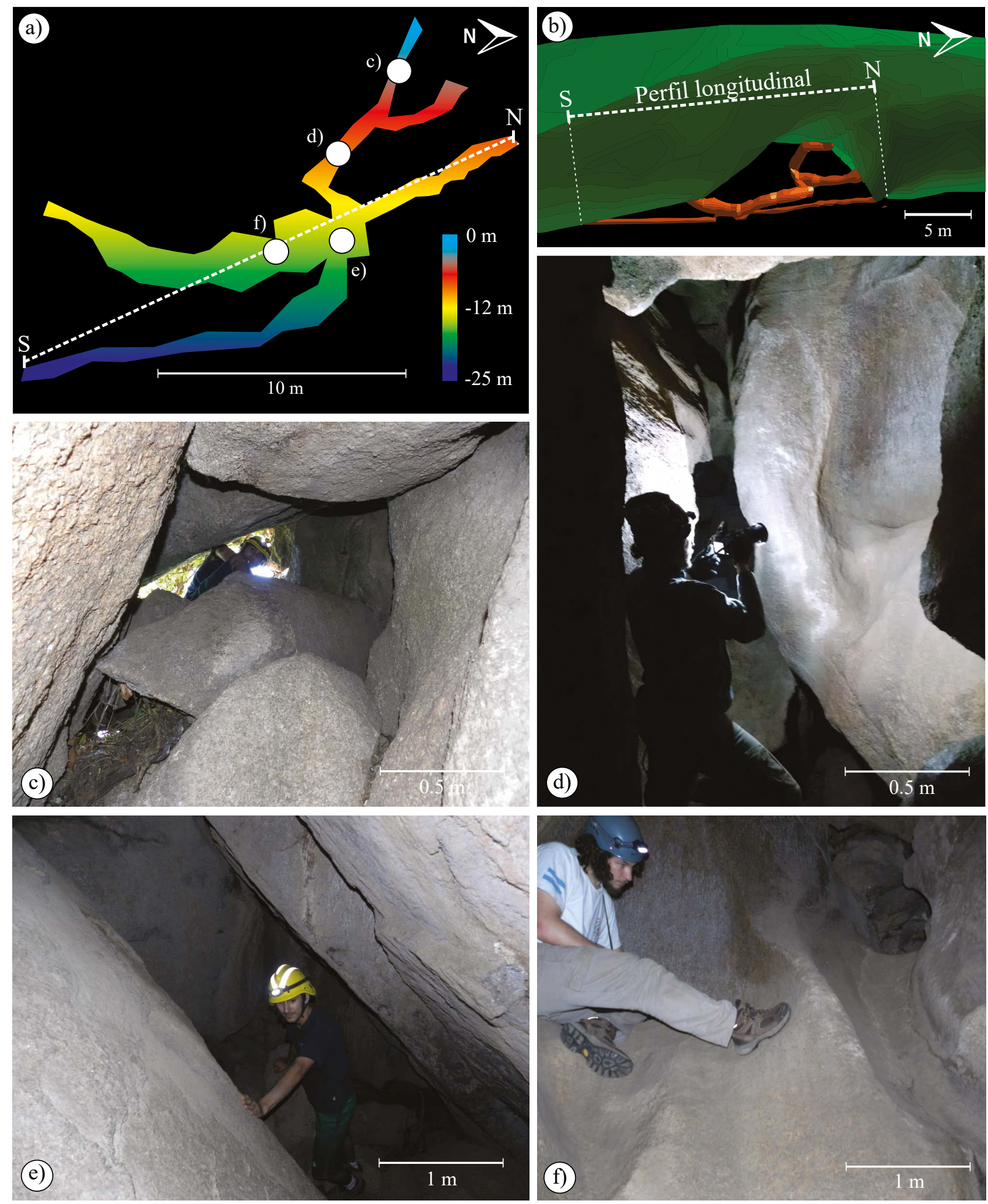

Figura 6. a) Sección vertical de la caverna. b) Modelo 3D de la caverna realizado con el software VisualTopo a partir de datos de poligonales abiertas. c) y e) Vistas del interior de la caverna en el sector Norte. d) y f) Vistas del interior de la caverna en el sector Sur. 


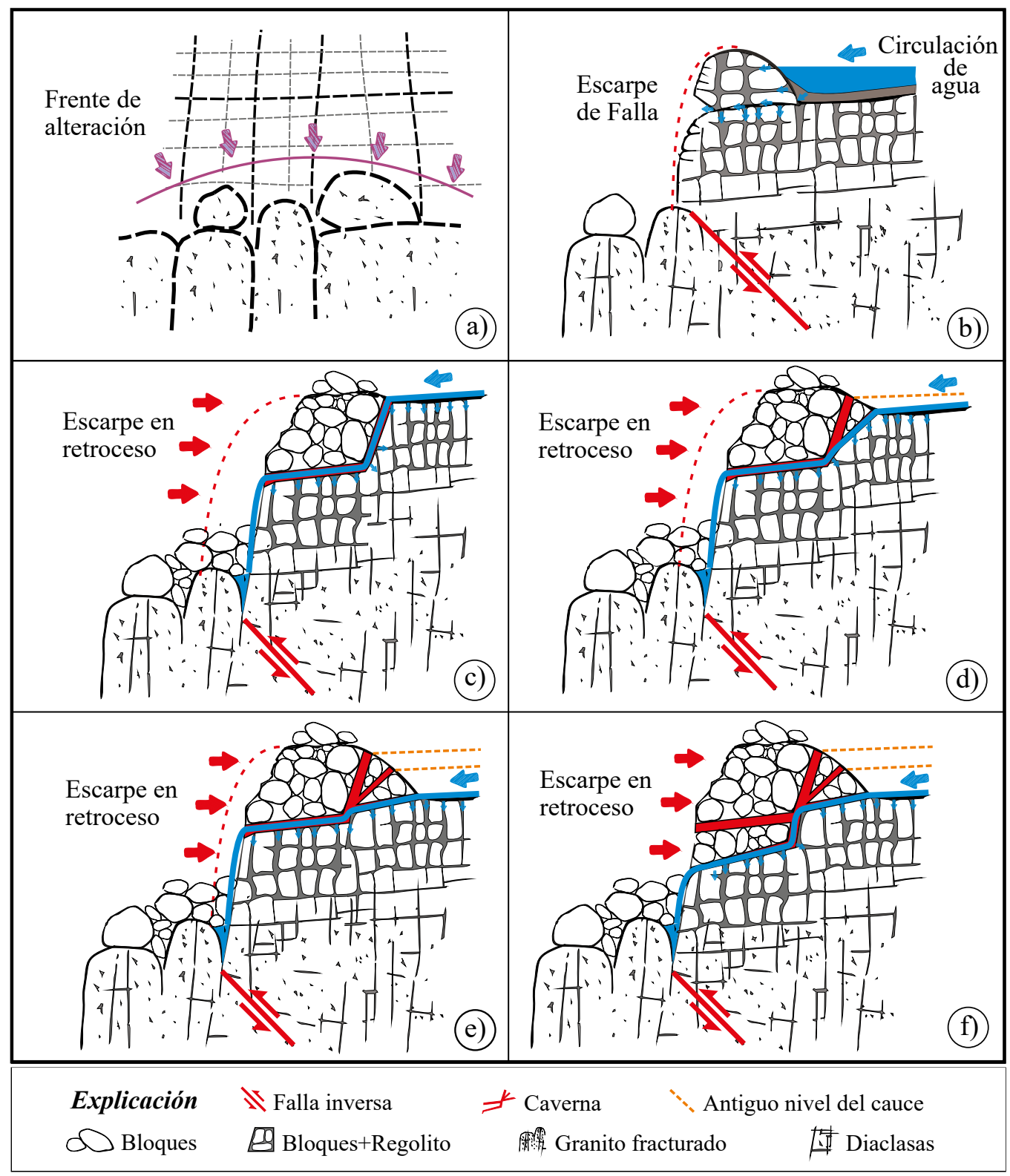

Figura 7. Modelo genético-evolutivo para la caverna del Río Subterráneo. a) Desarrollo de un perfil de meteorización profundo que condiciona la formación de un paleorrelieve con predominio de una morfología abochonada. b) Formación de la cuenca hidrográfica a partir de un escarpe mixto (erosivo y por falla). c, d, e y f) Formación de las galerías principales en sucesivas fases de erosión que remueven el material previamente alterado.

desmantelados hasta el frente mismo de corrosión, debido a un cambio en las condiciones ambientales hacia un clima progresivamente más seco durante el Cretácico, dejando en superficie porciones de roca más resistentes como relictos masivos (Carignano et al., 1999, 2014). Así es como los nubbins, castles koppies y bornhadts persistirían en el relieve general de la planicie, quedando como formas aisladas heredadas (residuales) de una etapa anterior (más húmeda que la actual) (Rabassa et al., 1996; Rabassa y Ollier, 2014; Crespi, 2015).

Continuando con la secuencia evolutiva, una segunda generación de discontinuidades formaría las estructuras latentes por concentración de cargas, que al ser expuestas se convertirían en bloques, tafoni y gnammas primarios endógenos (Vidal-Romani y Yepes, 2004; Crespi, 2015).

Respondiendo a un alzamiento general de la Sierra originada por la tectónica andina durante el Cenozoico (18-11 Ma) (Yañez et al., 2001; Ramos et al., 2002) y a la instalación de las condiciones climáticas actuales, se originarían las formas erosivas que responden a la instauración de los sistemas de escorrentías, tales como pilas o pilancones fluviales, regueros y acanaladuras. La última generación estaría compuesta por geoformas recientes o actuales, en donde la influencia de la alteración mecánica produciría bloques partidos, y la alteración química generaría las espeleotemas y el perfil de meteorización actual. Asociados con este último, se originarían las laderas zapadas o abrigos por ciclos sucesivos de meteorización-erosión.

Teniendo presente este esquema general, se hace evidente que el modelo propuesto para el origen de la caverna del Valle de Los Lisos, resulta consecuente con la historia evolutiva de los paisajes graníticos del Batolito de Achala y de las Sierras de Córdoba. 


\section{CONCLUSIONES}

El origen de la caverna del Valle de Los Lisos puede sintetizarse en tres etapas consecuentes con la historia evolutiva de los paisajes graníticos del Batolito de Achala: 1) Desarrollo de un perfil de meteorización profundo que condiciona la formación de un paleo-relieve con predominio de una morfología abochonada. 2) Generación, a partir de un escarpe mixto (erosivo y estructural), de la cuenca hidrográfica que alimenta el cauce que circula a través de la caverna. 3) Formación de las galerías principales en sucesivas fases de erosión.

La congruencia encontrada entre las observaciones realizadas tanto en el interior de la caverna como en su entorno geomorfológico, permiten comprender que estos ambientes subterráneos (al igual que otros accidentes geográficos y geoformas graníticas) no son caóticos, sino por el contrario, están estructurados por procesos geológicos y geomorfológicos a lo largo del tiempo, que responden a un marco evolutivo común para todo el macizo rocoso. De esta manera, el estudio de las cavidades del subsuelo se constituye en una pieza clave para comprender la historia geológica del paisaje en el que se encuentran.

De acuerdo con las observaciones efectuadas, para que se produzca el proceso de cavernamiento y sea efectivo, en este tipo de ambientes, deben coincidir al menos tres factores esenciales: disponibilidad de agua, diaclasado del macizo rocoso y una situación topográfica favorable.

\section{AGRADECIMIENTOS}

Los autores reconocemos el apoyo de IEYDAT CORP SRL, y en particular de Pablo Pistoia, Ana Lorena Brandolin y José Luis Amuchastegui, al facilitarnos el ingreso al sitio de estudio y colaborar en las campañas espeleológicas.

Además, hacemos llegar nuestro más sincero agradecimiento a los evaluadores, Dr. Theofilos Toulkeridis y otro anónimo, cuyos comentarios enriquecieron este trabajo. A la Glga. Micaela Pleitavino por la traducción al inglés del resumen. Al Consejo Nacional de Investigaciones Científicas y Técnicas (CONICET) y a la Facultad de Ciencias Exactas Físicas y Naturales de la Universidad Nacional de Córdoba (FCEFyN-UNC) por el apoyo financiero.

\section{REFERENCIAS}

Bates, R.L., Jackson, J.A., 1987, Glossary of Geology: Virginia, American Geological Institute, 788 pp.

Bense, F.A., Löbens, S., Dunkl, I., Wemmer, K., Siegesmund, S., 2013, Is the exhumation of the Sierras Pampeanas only related to Neogene flat-slab subduction? Implications from a multi-thermochronological approach: Journal of South American Earth Sciences, 48, 123-144.

Campbell, E.M, 1997, Granite landforms: Journal of the Royal Society of Western Australia, 80 (3), 101-112.

Campbell, E.M., Twidale, C.R., 1995, The various origins of minor granite Landforms: Cadernos do Laboratorio Xeolóxico de Laxe, 20, 281-306.

Capitanelli, R.G., 1979, Clima, en Vásquez, J.B., Miatello, R.A., Roqué, M.E. (eds.), Geografía Física de Córdoba: Córdoba, Boldt, 45-138.

Carignano, C., Cioccale, M., Rabassa, J., 1999, Landscape antiquity of the Central-Eastern Sierras Pampeanas (Argentina): geomorphological evolution since Gondwanic times: Zeitschrift fur Geomorphologie Supplementband, 245-268.

Carignano, C., Kröhling, D., Degiovanni, S., Cioccale, M., 2014, Geomorfología, en Martino, R., Guereschi, A. (eds.), Relatorio XIX Congreso Geológico Argentino: Geología y Recursos Naturales de la Provincia de Córdoba: Córdoba, Asociación Geológica Argentina, 747-822.

Cerutti, N., 2002, Geoformas graníticas en el Batolito de Achala, Sierras Grandes de la Provincia de Córdoba, Argentina: Córdoba, Universidad Nacional de Córdoba, trabajo final de grado, $114 \mathrm{pp}$.

Cioccale, M.A., Pasquini, A.I., Depetris, P.J, 2008, Hallazgo de espeleotemas silíceas en rocas graníticas del batolito de Achala, Sierras Pampeanas de Córdoba: Revista de la Asociación Geológica Argentina, 63(3), 417-420.

Crespi, M.F., 2015, Morfogénesis del paisaje granítico del batolito de Achala, provincia de Córdoba: análisis del control geológico: Córdoba, Universidad Nacional de Córdoba, trabajo final de grado, 199 pp.

Dorais, M.J., Lira, R., Chen, Y., Tingey, D., 1997, Origin of biotite-apatite-rich enclaves, Achala batholith, Argentina: Contributions to Mineralogy and Petrology, 130(1), 31-46.

Finlayson, B., 1981, Underground streams on acid igneous rocks in Victoria: Helictite, 19(1), 5-14.

Gaal, L., Bella, P., 2008, Granites and granite caves in the Western Carpathians: Cadernos do Laboratorio Xeolóxico de Laxe, 33, 11-18.

García-Aráoz, E., Cioccale M., 2015, Geoformas Graníticas: Distribución Espacial y Procesos Formadores. Batolito De Achala, Provincia De Córdoba (resumen), en VI Congreso Argentino de Cuaternario y Geomorfologia, Ushuaia: Ushuaia, Editorial Utopías, 274-275.

Gordillo, C.E., Lencinas, A.N., 1979, Sierras Pampeanas de Córdoba y San Luis, en Leanza, A.F (ed.), Geología Regional Argentina: Córdoba, Academia Nacional de Ciencias, 577-650.

Krapovickas, J.M., Tauber, A.A., 2016, Estratigrafía de las áreas cumbrales de las Sierras Pampeanas de Córdoba: geocronología, modelo regional, paleoambiente y paleoclima en una región poco conocida de Argentina: Revista Mexicana de Ciencias Geológicas, 33(1), 105-121.

Lazarte, S., 2002, Descripción topográfica de la cueva de Los Lisos, Los Gigantes, Provincia de Córdoba: Salamanca, 11, 32-35.

Lazaridis, G., Pipera, K, 2008, Preliminary report on granite caves in Greece: Cadernos do Laboratorio Xeolóxico de Laxe, 33, 101-113.

Löbens, S., Bense, F.A., Wemmer, K., Dunkl, I., Costa, C.H., Layer, P., Siegesmund, S., 2011, Exhumation and uplift of the Sierras Pampeanas: preliminary implications from $\mathrm{K}-\mathrm{Ar}$ fault gouge dating and low-T thermochronology in the Sierra de Comechingones (Argentina): International Journal of Earth Sciences, 100(2), 671-694.

Manzur, A., 1995, Aspectos genéticos de los suelos presentes en Atum Pampa y Athos Pampa, Sierras Grandes, Córdoba, Argentina: Córdoba, Argentina, Facultad de Ciencias Exactas, Físicas y Naturales, Universidad Nacional de Córdoba, Tesis Doctoral, 114 pp.

Martino, R.D., Guereschi, A.B., Carignano, C.C., 2012a, Influencia de la tectónica Preandina sobre la tectónica Andina: El caso de la falla de la Sierra Chica, Sierras Pampeanas de Córdoba: Revista de la Asociación Geológica Argentina 69(2), 207-221.

Martino, R.D., Toledo, A.E., Guereschi, A.B, 2012b, Lineamientos transtensionales en el margen noroccidental del batolito de Achala y su relación con la exhumación de rocas de alto grado y la extensión cretácica en las Sierras de Córdoba, en 15ºunión de Tectónica, Libro de Resúmenes: San Juan, Argentina Facultad de Ciencias Exactas Físicas y Naturales, Universidad Nacional de San Juan, 90.

Martino, R.D., Guereschi, A.B, Caro Montero, A., Abril, E.G., 2017, El control de la tectónica frágil Preandina en la estructura y morfología actual del Batolito de Achala, Sierras Pampeanas de Córdoba (resumen), en XX Congreso Geológico Argentino, Tucumán: San miguel de Tucumán, Simposio de Tectónica Pre-Andina, 105-107.

Montes, A., 1956, Cronología de nuestra prehistoria: Revista de la Facultad de Ciencias Exactas Físicas y Naturales, Universidad Nacional de Córdoba, $18(3-4), 1-41$.

Migon, P. (Ed.), 2006, Granite landscapes of the World: Gran Bretaña, Oxford University Press, $417 \mathrm{pp}$.

Ollier, C.D., Smith, J.M.B., 1994, Crombie's Cave. A granite cave in New England, NSW: Helictite, 32(1), 17-20.

Rabassa, J., Ollier, C. (Eds.), 2014, Gondwana Landscapes in Southern South America: Argentina, Uruguay and Southern Brazil: Dordrecht, Springer Science y Business Media, $561 \mathrm{pp}$.

Rabassa, J., Zárate, M., Cioccale, M., Carignano, C., Partridge, T.C., Maud, R., 1996, Paisajes relictuales de edad Gondwánica en áreas cratónicas de Argentina (resúmen), en $13^{\circ}$ Congreso Geológico Argentino y III Congreso de Exploración de Hidrocarburos, Buenos Aires: Buenos Aires, Asociación Geológica Argentina, (4), 219. 
Ramos, V., Cristallini, E.O., Pérez, D.J., 2002, The Pampean flat-slab of the central Andes: Journal South American Earth Sciences, 15, 59-78.

Rapela, C.W., Baldo, E.G.A., Pankhurst, R.J., Fanning, C.M., 2008, The devonian Achala batholith of the Sierras Pampeanas: F-rich, aluminous A-Type granites, en $6^{\circ}$ South American Symposium on Isotope Geology,: San Carlos de Bariloche, Actas, 8 p. (en disco compacto)

Roqué, C., Linares, R., Rodríguez, R., Zarroca, M., 2011, Granite caves in the north-east of the Iberian Peninsula: Artificial hypogea versus tafoni. Zeitschrift für Geomorphologie, 55(3), 341-364.

Scheuerer, M., Lundberg, J., Sjöberg, R., 2013, Gobholo cave: a long granite cave in Swaziland (Southern Africa) en 16th 16th International Congress of Speleology: Brno, Czech Republic, International Show Caves Association, Proceedings. 3, 305-307.

Sjöberg, R., 1969, A proposal for a classification system for Granitic Caves: Abh, 2(5), 25-28.

Song, Z., Zhou, J., Li, H., Liu, X., 2011, Discovery of especially large bubblelike miarolitic granites in Laoshan and its geological and geomorphic significance, en 2011 International Conference on Remote Sensing, Environment and Transportation Engineering (RSETE): Nanjing, China, IEEE, 3068-3071.

Song Z.J., Tang W.J., Liu X.Q., Wang L.B., Xiang S.B., Li J.P., Yang S.S., 2015, Genesis and geological significance of granite caves in Laoshan of China: Chemical Engineering Transactions, 46, 763-768.

Tauber, A.A.(h), Goya, F., 2006, Estratigrafía y fósiles del Pleistoceno-Holoceno de las pampas de altura en el departamento Cruz del Eje, Córdoba, Argentina, en III Congreso Argentino de Cuaternario y Geomorfología: Córdoba, Argentina, Actas de resúmenes, 2, 717-726.
Twidale, C.R., 2012, Granite landforms: Amsterdam, Elsevier, 371 pp.

Twidale, C.R., Vidal-Romaní, J.R., 2005, Landforms and geology of granite terrains: Amsterdam, Balkema, $352 \mathrm{pp}$

Vidal-Romaní, J. R., Yepes, J., 2004, Historia de la morfogénesis granítica: Cadernos do Laboratorio Xeolóxico de Laxe, 29, 331-360.

Vidal-Romaní, R.J., Sanjurjo Sánchez, J., Rodríguez, M., Fernández Mosquera, D., 2010, Speleothem development and biological activity in granite cavities: Geomorphologie: relief, processus, environnement, 16(4), 337-346.

Willems, L., Compère, P., Hatert, F., Pouclet, A., Vicat, J. P., Ek, C., Boulvain, F., 2002, Karst in granitic rocks, South Cameroon: cave genesis and silica and taranakite speleothems: Terra Nova, 14(5), 355-362.

Yañez, G., Ranero, G., Von Huene, R., Díaz, J., 2001, Magnetic anomaly interpretation across a segment of the Southern Central Andes $\left(32-34^{\circ} \mathrm{S}\right)$ implications on the role of the Juan Ferna'ndez Ridge in the tectonic evolution of the margin during upper Tertiary: Journal of Geophysical Research, 106, 6325-6345.

Manuscrito recibido: julio 26, 2017

Manuscrito corregido recibido: diciembre 4, 2017

Manuscrito aceptado: diciembre 4, 2017 